\title{
Acoustically elicited stapedial reflex in cochlear implanted patients
}

\author{
Petrov SM* \\ PE, St. Petersburg, Russia
}

\begin{abstract}
The results of registration of stapedial reflex in a response to electrical stimuli are often used during the fitting process of cochlear implant. Usually, the most comfortable loudness (MCL) levels of the working program are higher than threshold levels of the stapedial reflex. It is interesting to look at the reflex from another point of view, namely, to investigate the stapedial reflex in response to acoustic stimuli of low, medium and high frequencies in a group of seven experienced children who had been using their speech processor for three or more years. It was found that threshold sound pressure levels of the reflex to sound stimuli are within a normal range. The reflex threshold levels were found to underestimate the behavioral MCL values and therefore should be used with caution during the first fitting sessions.
\end{abstract}

\section{Introduction}

The method of registration of the stapedial reflex in a response to electrical stimuli are used in the fitting of cochlear implant. But as a rule, the most comfortable loudness (MCL) levels of the working program are higher than threshold levels of the stapedial reflex $[1,2]$. It is interesting to look at the reflex from the opposite point of view, namely, to investigate the stapedial reflex on sound stimuli in experienced implanted patients configured optimally. The aim of this study is the investigation of the stapedial reflex in a response to acoustic stimuli of low, medium and high frequencies.

\section{Materials and methods}

7 smart implanted patients participated in this study. Age 7-14 years. All patients used the Med-El 12-channel implant "Concerto". They came to the 4 th or later fitting session. Everyone had been optimally fitted and everyone deliberately chose a work program from four programs of increasing loudness. On repeated visits, patient working programs were not changed over the past few years. All patients were familiar with the audiologist and participated with interest in the study. The investigation was conducted at working (comfortable) for each patient program which was selected in accordance with our instruction-explanation [3].

Since this is the first study of the stapedial reflex on sound stimuli in implanted patients, the issues of the influence of gender, age, etiology of deafness, side of the operation, etc. are beyond the scope of this study. The main requirements for selecting children to participate in the experiment were: a clear choice of a comfortable program, a desire to cooperate, a normal tympanogram, and the presence of a stapedial reflex. The parameters of the tympanogram (compliance and pressure) in all children were in the normal ranges.

Sine wave stimuli were generated using the "Adobe Audition" program. The parameters of acoustic stimulus were the following: duration is $260 \mathrm{~ms}$; pulse decay time and pulse rise time of stimuli were $5 \mathrm{~ms}$; frequencies were 379,1123 and $4365 \mathrm{~Hz}$ - the central frequencies of the 2nd, 6th and 11th channels of the cochlear implant, respectively.
We used a sequence of 10 stimuli of each frequency. This sequence was created in the "Adobe Audition"program. The intervals between the leading edge of the tone pulses are 1s, intensity levels of sequential pulses are increased with step of $4 \mathrm{~dB}$. The stimuli were amplified by an Azur640A amplifier so the intensity of successive stimuli increased from 70 to $106 \mathrm{~dB}$ SPL. Sound pressure levels were calibrated in an artificial ear 4153 of "Bruel\&Kjar". Patients listened to stimuli of $106 \mathrm{~dB}$ SPL without any negative reaction.

We switched on a speech processor at working program and placed it under the circumaural embouchure of headphone TDH-3 through which we presented a sound stimuli. Antenna of an implant connected to a long wire was placed on the patient's head. The studies were conducted on the optimal program of each child.

Acoustic stapedial reflexes were registered on the contralateral ear using an audiometer-impedance meter AA220 of "Interacoustics". Impedance meter worked in the reflex decay mode. We simultaneously switched on sound stimulation and the reflex decay test of an impedance meter.

As an example, Figure 1 shows the result of one registration of stapedial reflex printed from the impedance audiometer screen.

As can be seen from the figure, the amplitude of stapedial reflex is linearly increasing with the increase of SPL of acoustic stimuli. The threshold level of a stapedial reflex is $94 \mathrm{~dB}$ SPL. The maximum amplitude of stapedial reflex was at $106 \mathrm{~dB}$ SPL.

It is obvious that the threshold level of the reflex is lower than an electrical MCL level recorded in this channel. The results of all patients were registered in the same manner. The results of registration of the

${ }^{\star}$ Correspondence to: Petrov SM, PE, St. Petersburg, Russia, Tel: +79117495446; E-mail: senn2001@mail.ru

Key words: cochlear implant, fitting, most comfortable loudness level, sound stimuli, threshold level of stapedial reflex

Received: September 15, 2020; Accepted: September 25, 2020; Published: October 02, 2020 
stapedial reflex were printed on the printer of the impedance meter, scanned and then transferred into digital form using the program "Grafula".

\section{Results and discussion}

The Figure 2 shows the results of measurements of the amplitude of the stapedial reflex depending on the sound pressure level of acoustic stimuli of $1123 \mathrm{~Hz}$ frequency in seven implanted patients.

As can be seen from the graphs there is a wide range of individual values of the threshold levels of the stapedial reflex on sound stimuli in the examined patients. The threshold levels of the stapedial reflex are in the range of $81-97 \mathrm{~dB}$ SPL, the average value is $88.4 \pm 5.4 \mathrm{~dB}$ SPL. It should be noted that the amplitude of the reflex increases linearly with the increase of the intensity of acoustic stimuli from the threshold levels of reflexes to the maximum values at intensity level of $106 \mathrm{~dB}$ SPL. Since the reflex and loudness are connected, this means that loudness of input stimuli increases continuously from the threshold intensity of reflex to $106 \mathrm{~dB}$ SPL. At level of sound of $106 \mathrm{~dB}$ SPL subjects had no negative reaction to single stimulus of $260 \mathrm{~ms}$ duration.

A similar pattern of a wide range of reflex threshold values and a linear increase of the amplitude of reflexes from threshold levels to the maximum SPL of a stimuli is observed in low and hi gh frequency channels too.

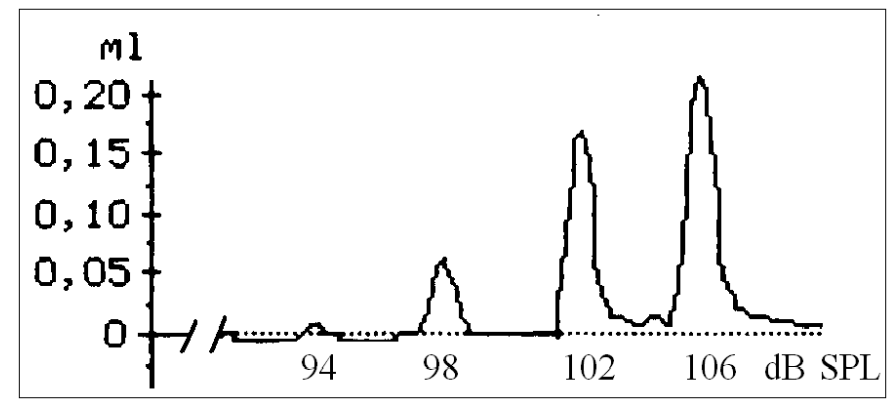

Figure 1. The amplitude of the stapedial reflex depending on the sound pressure level of acoustic stimuli in a patient K. Frequency of stimuli is $1123 \mathrm{~Hz}$. Printout image from the screen of an impedancemeter.

The abscissa axis is the sound pressure level of the stimuli, dB SPL.

The ordinate axis is the compliance of a drum membrane (amplitude of stapedial reflex), $\mathrm{ml}$

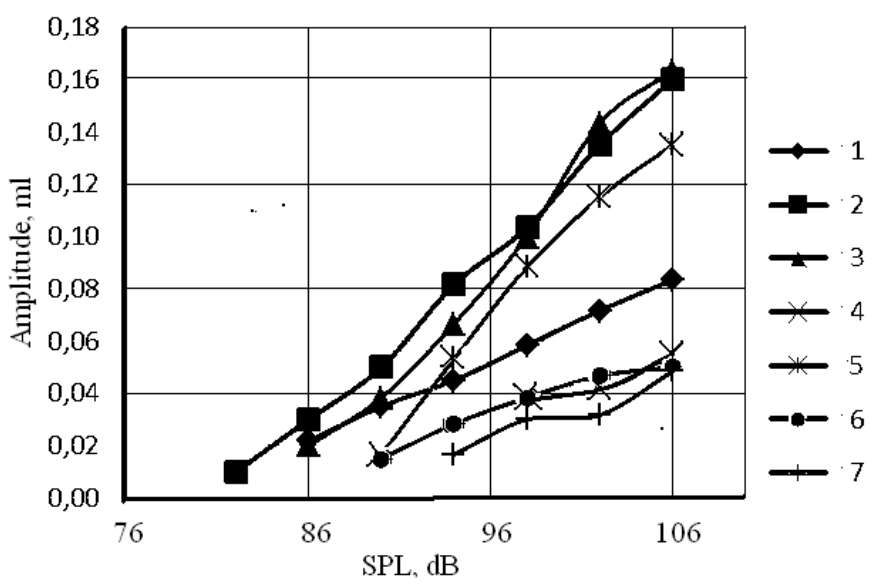

Figure 2. The dependence of the amplitude of the stapedial reflex $(\mathrm{ml})$ on the sound pressure level (dB SPL) of acoustic stimuli of 1123 frequency $\mathrm{Hz}$ in seven implanted patients.

The abscissa axis - the sound pressure level of the stimuli, dB SPL.

The ordinate axis - amplitude of the stapedial reflex, $\mathrm{ml}$
Table 1. Ranges of individual results of measurements of threshold levels of stapedial reflex on tone signals of three frequencies and average values of the threshold levels of reflex in three channels of $\mathrm{Cl}$

\begin{tabular}{|c|c|c|}
\hline Frequency, Hz & Threshold range, $\mathbf{d B}$ & Mean,dB \\
\hline 379 & $85-101$ & $90.7 \pm 6.0$ \\
\hline 1123 & $81-97$ & $88.4 \pm 5.4$ \\
\hline 4365 & $73-85$ & $81.8 \pm 4.6$ \\
\hline
\end{tabular}

The results of measurements are presented in the Table 1.

The ranges of individual results are similar in all channels - 12$16 \mathrm{~dB}$. Mean values of threshold levels of the stapedial reflex on tonal stimuli of three frequencies in CI patients are located in the range of $81.8-90.7 \mathrm{~dB}$ SPL. It should be noted that these average values of threshold levels in CI patient coincide with the threshold levels of reflex in subjects with normal hearing. In normal subjects the average values of the threshold levels of the stapedial reflex on tonal stimuli are in the range of 80 to $90 \mathrm{~dB}$ SPL [4,5].

It should be noted that reflexes were recorded in all channels of an implants at the working programs of all children. All parents chose the working program from four MAPs recorded in the processor memory. Consequently, all of the MCL levels established in the working programs are above the threshold levels of reflex. Individual results of threshold levels of the stapedial reflex are located in the range from 73 to $101 \mathrm{~dB}$ SPL. In normal subjects the individual values of the threshold levels of stapedial reflex on tonal stimuli are in the range of 70-100 dB SPL [6,7].

It is obvious that if the average values of the reflex thresholds in a normal subject are from 80 to $90 \mathrm{~dB}$ SPL (Gelfand, 2004) the individual results of the subjects are in a wider intensity range. It means that the threshold levels of stapedial reflexes in CI patients are in the normal range.

As an example, we will discuss the results of measurements in a patient whose threshold level of the stapedial reflex at a frequency of $4365 \mathrm{~Hz}$ equals $73 \mathrm{~dB}$ SPL. It is obvious that the reflex threshold is registered at the current level much less than the subjectively set MCL level in the working program (further initial program). This child uses 3rd program as working (everyday) one. Parents chose this program in accordance with our explanation-recommendation: "The program is optimal one if your child sometimes hears loud sounds" [3]. This quickwitted child himself said that 4th program was slightly loud one; the 2nd program was a quiet one. A child himself asks to switch on the $3 \mathrm{rd}$ program.

Consider what happens if we set the current levels at which the threshold stapedial reflex is registered as MCL level in a new program. In the new program, as well as in the initial, the speech processor will process the input audio signal in the intensity range of 40-106 dB SPL, but the patient's perception will be different than with the initial program.

Naturally, in the new program the reflex will be registered only at the sound level of $106 \mathrm{~dB}$ SPL, at $73 \mathrm{~dB}$ SPL it will not be, i.e. on the new program at an input signal of $106 \mathrm{~dB}$ SPL the patient will hear the same loudness as at $73 \mathrm{~dB}$ SPL of the initial program. Consequently, the new program will unnecessarily reduce the maximum loudness achieved at the new MCL level.

Further, if we set the threshold current level in the new program as $10 \%$ of the MCL levels, the processor will produce this current level at the trigger level of sound equals to $40 \mathrm{~dB}$ SPL, but the signal with this current level will not be heard, because the threshold current in the new program ( $10 \%$ of the new MCL level, equal to the threshold level 
of the stapedial reflex) will be below the threshold of audibility. The real threshold of a current audibility is $10 \%$ of the MCL levels in the initial program. That is, some part of the sound range that the patient perceived on the initial program will not be heard on the new program. Let's say that in accordance with the output compression function ('maplaw'), the threshold level of the current recorded in the initial program is reached at $45 \mathrm{~dB}$ SPL in the new program.

Thus, in the new program, the input sound in the range of 40-106 $\mathrm{dB}$ SPL will be heard from $45 \mathrm{~dB}$ SPL and $106 \mathrm{~dB}$ SPL will be equal in loudness to that the patient heard at $73 \mathrm{~dB}$ SPL on the initial program. Clearly quieter than the initial program. Therefore, when setting MCL levels as reflex thresholds levels, there will be an unjustified increase in the threshold of sound perception from 40 to $45 \mathrm{~dB}$ SPL and a significant decrease in loudness at a maximum input intensity of 106 dB SPL. It is obvious that such changes will cause loss of input signal information, which will lead to deterioration of speech intelligibility. This is a speculative reasoning which does not require verification. The patient himself, after assessing the perception of speech with a new setting of MCL levels as the threshold levels of reflex, will ask to return the previous setting and will not accept the program with MCL levels equal to the reflex threshold levels. We wrote more about the impact of the threshold and MCL settings earlier [8,9].

Similarly, we can consider the rest of our results. All individual values of the reflex thresholds are in the intensity range from 73 to $101 \mathrm{~dB}$ SPL. Therefore, the reflex threshold levels are recorded at SPL less than $106 \mathrm{~dB}$ SPL, i.e. threshold current levels of reflexes are less than MCL levels established by the patient subjectively in the working program. Consequently, the threshold levels of the stapedial reflex to electrical stimuli are not used as the MCL levels of the working (optimal) program. A reasoning similar to the above with respect to the threshold level of reflex $73 \mathrm{~dB}$ SPL can be applied to all our results obtained by measuring the reflex threshold to a sound.

Our results on the threshold levels of a reflex below MCL levels coincide with many psychoacoustic literature data. For example, in the study of M. Bresnihan "was found that the C-level obtained with ESRs to be consistently lower across all electrodes. Of the 391 measurements made behavioral MCL levels exceeded the threshold levels of reflexes in 380 cases" [1]. Similar results were obtained by us [2] and were confirmed in many other studies.

Based on these studies, we agree with those authors who state that reflex thresholds can be used as a basis for conditioning behavioral responses [10] or that threshold values of stapedial reflexes may usefully assist in programming the CI [11].

An indirect argument against the use of threshold levels of reflex as MCL levels is that almost all of our patients (more than one thousand, with a few exceptions) participated in the SWEEP stimulationregistration of the stapedial reflex without any negative reactions [12].

Answer, please, such question. How can the MCL levels setting being equal to the reflex threshold levels be the final result of the fitting, if the maximum loudness of sound that the patient perceives on the new program is achieved with an input sound intensity of $73 \mathrm{~dB}$ SPL at the initial program?

It should be borne in mind that the high correlation between MCL levels and reflex threshold levels is also not an argument in favor of the final MCL levels setting on a level of reflex thresholds. The correlation coefficient estimates only the direction and strength of the coupling between these parameters but does not give any indication of numerical values as far as the current values of the reflex thresholds are greater or lower than the MCL levels.

In conclusion we can draw some parallels between CI and 1-st stage sensorineural hearing loss of peripheral type:

- Neural origin of hearing losses in both cases

- In both cases a hearing loss is $40 \mathrm{~dB}$

- At 106 dB SPL patients hear loud sounds (near tolerance level)

- The threshold sound pressure levels of the stapedial reflex are within normal limits.

- The perceived loudness of input stimuli is increased linearly from the threshold levels of reflex to $106 \mathrm{~dB}$ SPL.

There is possible similarity with the results of Fowler test [13].

With such a combination of features diagnosed in patients with SNHL, the presence of loudness recruitment is observed. We can say that CI patients also have a similar phenomenon.

\section{Conclusions}

1. The mean values of the threshold sound pressure levels of the stapedial reflex to acoustic stimuli of three frequencies in implanted patients are in the normal range ( $81.8-90.7 \mathrm{~dB} \mathrm{SPL})$, and the individual results are in the normal range of $70-100 \mathrm{~dB}$ SPL.

2. The dependence of the amplitude of the stapedial reflex on the SPL of the sound signal is linear in the range of the intensity of the tonal stimuli from the threshold level of the reflex to $106 \mathrm{~dB}$ SPL.

3. The current threshold levels of the stapedial reflex to sound stimuli are below the subjective MCL levels and they cannot be used as the most comfortable loudness levels in the vast majority of implanted patients.

\section{References}

1. Bresnihan M, Norman G, Scott F, Viani L (2001) Measurement of comfort levels by means of electrical stapedial reflex in children. Arch Otolaryngol Head Neck Surgery 127: 963-966. [Crossref]

2. Petrov SM, Shchukina AA (2007) Objective methods of fitting speech processors of cochlear implants Combi-40/40+ and Tempo+: Impedance Technique. Vestnik Otorinolaringology 5: 20-22. [Crossref]

3. Petrov SM, Tsjuk AA (2015) Instruction for audiologists and cochlear implanted patients. ISBN 3: 659-699.

4. Reker U (1977) Normal values of the ipsilateral acoustic stapedius reflex threshold Arch Otorhinolaryngol 215: 25-34

5. Gelfand S (2004) Hearing: An Introduction to Psychological and Physiological Acoustics. 5th Edition

6. Lopotko AI (1977) Some characteristics of the acoustic muscle reflex in the normal and in the pathology of the organ of hearing. Journal Aural Nose and Throat Diseases 2: $39-41$.

7. Altman JA, Tavartkiladze GA (2003) Manual of audiology. DMK Press.

8. Petrov SM, Gritsjuk MI (2016) Fitting of the cochlear implant in child: Sweep and SHCHUP. Austin J Otolaryngol 3(4): 1-3

9. Petrov SM (2017) The threshold problem in implant patients. Global Journal of Medical Research 17: 21-23.

10. Hodges AV, Butts S, Dolan-Ash S, Balkany TJ (1999) Using electrically evoked auditory reflex thresholds to fit the CLARION cochlear implant. Ann Otol Rhinol Laryngol Supplement 177: 64-68. 
11. Guida M, Falcioni M, Di Lella F, Negri M, Fagnani E, et al. (2019) Multielectrode sequential versus single electrode stimulation to elicit the stapedial reflex during cochlear implantation: Correlation with maximum comfort level. Eur Ann Otorhinolaryngol Head Neck Dis 136: 169-172. [Crossref]
12. Petrov SM (2017) Practical implementation of the SWEEP-session of stimulationregistration in CI fitting. Advanced Treatments in ENT Disorders 1: 13-15.

13. Fowler E (1937) The diagnosis of diseases of the neural mechanism of hearing by the aid of sounds well above threshold. Laryngoscope 47: 289-300.

Copyright: (C2020 Petrov SM. This is an open-access article distributed under the terms of the Creative Commons Attribution License, which permits unrestricted use, distribution, and reproduction in any medium, provided the original author and source are credited. 